\title{
STUDYING THE STABILITY BY USING LOCAL LINEARIZATION METHOD
}

\author{
Abdulghafoor Jasim Salim ${ }^{1}$, Kais Ismail Ebrahem², Suhirman ${ }^{3}$ \\ ${ }^{1)}$ College of Computer Science and Mathematics University of Mosul. \\ ${ }^{2}$ College of Education for pure science University of Mosul. \\ ${ }^{3)}$ Department of Information Engineering, University of Technology Yogyakarta \\ drabdul_salim@uomosul.edu.iq, kaisismail@yahoo.com,suhirman@uty.ac.id
}

\begin{abstract}
In this paper we study the stability of one of a non linear autoregressive model with trigonometric term by using local linearization method proposed by Tuhro Ozaki .We find the singular point ,the stability of the singular point and the limit cycle. We conclude that the proposed model under certain conditions have a non-zero singular point which is a asymptotically salable ( when $N^{t} \rightarrow 0$ ) and have an orbitaly stable limit cycle. Also we give some examples in order to explain the method.
\end{abstract}

Key Words : Non-linear Autoregressive model; Limit cycle; singular point; Stability.

\section{INTRODUCTION}

Most of the phenomenon have a non linear behavior, in the field of discrete time non linear time series modeling, there are different type of a non linear models such as bilinear model [1],[2], exponential autoregressive model [3], Threshold model [4] and so on.

The linear time series autoregressive models are stationary ( stable) if all the root of the characteristic polynomial lie inside the unit circle .In non linear time series models the researchers studying the stability by different methods [1]. Study the stability of non linear time series model [5] study the dynamic system with stability of nonlinear time series. Propose a dynamical approach for the stability of exponential autoregressive models [6], this approach is a local linearization technique used to find the approximated linear autoregressive model near the nonzero singular point of the model and he find the stability condition of a limit cycle if it exist. Study the local linearization of stochastic differential equation [7].

In this paper we study the stability to a first order non linear autoregressive model with trigonometric term by using the local linearization method proposed by [8]. By studying the stability of the singular point and limit cycle. Also we give some example to explain the method.

\section{RELATED WORKS AND METHOD}

\section{Definition 1: ( Time series):}

A time series is a set of observation measured sequential through time. These measurement may be made continuously or at a discrete set of time points [9].

Definition 2: (AR(p)):

The autoregressive model of order $p$ denoted by $\mathrm{AR}(\mathrm{p})$ is satisfying the following equation :

$$
\begin{gathered}
X_{t}+a_{1} X_{t-1}+\ldots . .+a_{p} X_{t-p}=Z_{t} \\
\text { Or } \quad X_{t}=f\left(X_{t-1}, \ldots, X_{t-p}\right)+Z_{t}
\end{gathered}
$$

Were $\mathrm{f}($.$) is some linear function, \left\{Z_{t}\right\}$ is a sequence of independent and identically distributed (IID) random variables ( white noise) and $a_{i}, \quad \mathrm{i}=1, \ldots, \mathrm{p}$ are constant [10]. A nay nonlinearity in $\mathrm{f}($.) results in a non linear mode.

\section{Definition 3: ( Exponential autoregressive model):}

The exponential autoregressive model of order $p$, $\operatorname{EXPAR}(p)$ is defined by the following equation : 


$$
X_{t}=\sum_{i=1}^{p}\left(\phi_{i}+\pi_{i} e^{-X_{t-1}^{2}}\right) X_{t-i}+Z_{t}
$$

Where $\phi_{i}, \pi_{i} \quad \mathrm{i}=1, . . \mathrm{p}$ are constants and $\left\{Z_{t}\right\}$ is a white noise.[3].

\section{Definition 4: (singular point):}

A singular point $\xi$ is defined as a point which every trajectory of

$$
X_{t}=f\left(X_{t-1}, X_{t-2}, \ldots, X_{t-p}\right) \text { beginning }
$$

sufficiently near it approach either for $t \rightarrow \infty$ or for $t \rightarrow-\infty$, if it approaches it for $t \rightarrow \infty$ we call it stable singular point other wise we call it unstable singular point. Obviously a singular point $\xi$ satisfies $\xi=\mathrm{f}(\xi)$ [8].

\section{Definition 5: (limit cycle):}

A limit cycle of $X_{t}=f\left(X_{t-1}, X_{t-2}, \ldots, X_{t-p}\right)$ is defined as an isolated and close trajectory $X_{t+1}, X_{t+2}, \ldots, X_{t+q} \quad$ where $\mathrm{q}$ is a positive integer. Closed means that if initial values $\left(X_{1}, X_{2}, \ldots, X_{p}\right)$ belong to the limit cycle then

$$
\left(X_{t+k q}, X_{2+k q}, \ldots, X_{p+k q}\right)=\left(X_{1}, X_{2}, \ldots, X_{p}\right)
$$

for any integer $\mathrm{k}$. isolated means that every trajectory begin sufficiently near the limit cycle approach either for $t \rightarrow \infty$ of for $t \rightarrow-\infty$. If it approach it for $\quad t \rightarrow \infty \quad$ we call it stable limit cycle and if it reaches it for $t \rightarrow-\infty$ we call it unstable limit cycle [8].

\section{Theorem (1);}

Let $\left\{X_{t}\right\}$ be expressed by the exponential model (2), the limit cycle $X_{t+1}, X_{t+2}, \ldots, X_{t+q}$ of period q of the model is orbital stable if $\left|\frac{\xi_{t+q}}{\xi_{t}}\right|<1$.

For the prove see [8].

\section{Theorem (2);}

Let $\left\{X_{t}\right\}$ be expressed by the exponential model (2), the limit cycle $X_{t+1}, X_{t+2}, \ldots, X_{t+q}$ of period $\mathrm{q}$ of the model is orbital stable when all the Eigen values of the matrix $\mathrm{A}=A_{q} . A_{q-1} \ldots A_{1}$, have absolute value less than one, where

$$
\mathrm{A}=\left[\begin{array}{c}
\phi_{1}+\left\{\pi_{1}-2 \sum_{j=1}^{p}\left(\pi_{j} x_{t+i-j}\right) x_{t+i-j}\right\} e^{-x_{t+i-j}^{2}} \\
1 \\
0 \\
\cdot \\
0
\end{array}\right.
$$

For the prove see [7].

\section{Definition 6:NAR(1): The proposed mode:}

The first order non-linear autoregressive model (the proposed model ) is satisfy the following equation :

$$
X_{t}=\left(\alpha+\beta \tan \left(2 \pi X_{t-1}\right)\right) X_{t-1}+Z_{t}
$$

Where $\left\{Z_{t}\right\}$ is a white noise process, and $\alpha$ , $\beta$ constants not equal to zero.

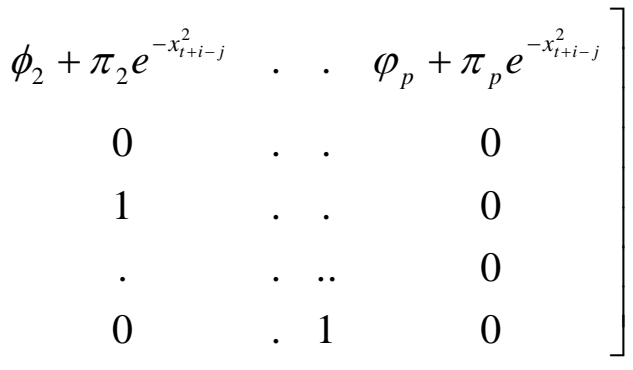

\section{RESULTS AND DISCUSSION}

\section{The stability of the proposed model:}

In this section, we shall study the stability of the first order non linear autoregressive model ( proposed model ) by using the local linearization method of T.Ozaki.

\section{Local linearization method:}

When we use the local linearization method which proposed by Ozaki there are three main steps namely : 
Step one: Find the non-zero singular point( if it exist) by using the definition such that $\xi=\mathrm{f}$ ( $\xi$ ) in the given model.

\section{The Singular point :}

To find the singular point of the model (3) we use the definition (4) of singular point and suppose that the white noise is not effected, that is $\left(Z_{t}=0\right)$

Then

$\xi=(\alpha+\beta \tan (2 \pi \xi) \xi$

$\xi=0$ zero singular point, let $\xi \neq 0$, then

The non zero singular point is :

$$
\xi=\frac{1}{2 \pi} \tan ^{-1}\left(\frac{1-\alpha}{\beta}\right): \beta \neq 0
$$

expansion for $\tan ($.$) function also suppose$ that

$\left|\xi_{i}\right|^{n} \rightarrow 0$ for $\mathrm{n} \geq 2$, also put $\left(Z_{t}=0\right)$ then we get

$\xi_{t}=M+N \xi_{t-1}$

Where

$\mathrm{M}=((\alpha-1) / 2 \pi)\left(\tan ^{-1}\left(\frac{1-\alpha}{\beta}\right)\right.$

And

Limit cycle (the stability):

Let the limit cycle of period q of the proposed model has the form:

$X_{t}, X_{t+1}, \ldots ., X_{t+q}=X_{t}$. the point $X_{s}$ near
Step two :Explain and find the stability condition to the singular point .

Step three: Find the limit cycle ( if it exist ) and then find the stability condition to the limit cycle for the model.

\section{The Stability condition for non-zero singular point:}

To find the stability condition for the non zero singular point $\xi$ of the model (7), we consider the variation difference equation in the neighborhood of $\xi$ by

Putting $\xi_{t-i}+\xi=X_{t-i} \quad, \mathrm{i}=0, \quad 1$. in the proposed model where $\left|\xi_{i}\right|$ is sufficiently very small and by using maclaurin series

$\mathrm{N}=\left[1+\frac{1-\alpha}{2 \pi} \tan ^{-1}\left(\frac{1-\alpha}{\beta}\right)\right]$

Equation (5) is a first order difference equation, which has the general solution

$\xi_{t}=N^{t} \xi_{0}+\frac{M\left(N^{t}-1\right)}{(N-1)} \quad, \mathrm{N} \neq 1$

The non-zero singular point to represent an asymptotically stationary process when the complementary function must decay to zero as $\mathrm{t} \rightarrow \infty$, that is if $N^{t} \rightarrow 0$ and this will happen when $\mathrm{N}<1$.

$X_{s}=X_{s}+\xi_{s}$, then replaced $X_{t}$ and $X_{t-1}$ by $X_{t}+\xi_{t}, X_{t-1}+\xi_{t-1}$ respectively and supposed that the white noise is not effected, then we have

the limit cycle is represented as

$X_{t}+\xi_{t}=\left[\alpha+\beta\left\{\tan \left(2 \pi\left(X_{t-1}+\xi_{t-1}\right)\right\}\left(X_{t-1}+\xi_{t-1}\right)\right]\right.$

By using Taylor series expansion and simple algebraic method,

and also suppose that $\left|\xi_{i}\right|^{n} \rightarrow 0$ for $\mathrm{n} \geq 2$, we get

$\xi_{t}=\left[\alpha+\beta\left\{\tan \left(2 \pi X_{t-1}\right\}\right] \xi_{t-1}\right.$
Equation (9) is a first order difference equation with periodic coefficient which is difficult to solve analytically. What we require is to know whether $\xi_{t} \quad$ converge to zero or not as 
$\xi_{t} \rightarrow 0$ and this can be checked by seeing whether the ratio $\left|\frac{\xi_{t+q}}{\xi_{t}}\right|<1$

(from the theorem(1)).

Since $\xi_{t}=\left[\alpha+\beta\left\{\tan \left(2 \pi X_{t-1}\right\}\right] \xi_{t-1}\right.$

Then

$$
\xi_{t+q}=\prod_{i=1}^{q}\left[\alpha+\beta\left\{\tan \left(2 \pi X_{t-1}\right\}\right] \xi_{t}\right.
$$

Therefore the limit cycle orbitaly stable if $\left|\frac{\xi_{t+q}}{\xi_{t}}\right|<1$, that is the absolute value of

$\left(\prod_{i=1}^{q}\left[\left\{\alpha+\beta\left\{\tan \left(2 \pi X_{t-1}\right\}\right]\right)\right.\right.$ is less than one

Example (1): let the time series $\left\{X_{t}\right\}$

modeled by

$=(0.4 * 0.00344 * 0.0011 * 0.0003 * 0.0001 *$

$0.00003)<1$, that is the limit cycle is orbitally stable.

Example (2): let the time series $\left\{X_{t}\right\}$

modeled by

$$
X_{t}=\left[1.2-0.2 \tan \left(2 \pi X_{t-1}\right)\right] X_{t-1}+Z_{t}
$$

The non-zero singular point is $\xi=\frac{1}{2 \pi} \tan ^{-1}\left(\frac{1-1.2}{-0.2}\right)$, that is $\xi=1.2337$, and $\mathrm{N}=0.975$ which satisfy that $\mathrm{N}<1$ and then the nonzero singular point is stable . The show that the limit cycle of period 6 to equation (12) orbitaly stable, we have

$$
\left(\prod_{i=1}^{6}\left[1.2-0.2 \tan \left(2 \pi x_{t+i-1}\right)\right] x_{t+i-1}\right.
$$

$=(\quad 0.3 * 0.5447 * 0.6222 * 0.6265$

$* 0.62412 * 0.6255)=0.02487$ which is less than one and hence the model has stable limit cycle.

\section{CONCLUSION}

In this paper, we study and find the stability conditions of the first order nonlinear autoregressive model by using a dynamical approach due to Ozaki. Which is a local

$$
X_{t}=\left[0.3+0.4 \tan \left(2 \pi X_{t-1}\right)\right] X_{t-1}+Z_{t}
$$

Then this model have zero singular point ( $\xi=0)$ and the non-zero singular point $\xi=\frac{1}{2 \pi} \tan ^{-1}\left(\frac{1-0.3}{0.4}\right)$, then the non- zero singular point is $0.167375471 \mathrm{rad}$.

And since $\mathrm{N}=\left[1+\frac{1-\alpha}{2 \pi} \tan ^{-1}\left(\frac{1-\alpha}{\beta}\right)\right]$.

Then $\mathrm{N}=1.1172>1$, that is the non -zero singular point is unstable.

And the limit cycle for equation (10) of period 6 which is $(0.4,0.00344,0.0011,0.0003$, $0.0001,0.0003$, and 0.4 ) by easy calculation

$$
\left(\prod_{i=1}^{6}\left[0.3+0.4 \tan \left(2 \pi x_{t+i-1}\right)\right] x_{t+i-1}\right.
$$

Also we can use theorem(2) to explain the stability of the limit cycle for the model (12), we get

$$
\begin{aligned}
& A_{1}=\left[\begin{array}{ll}
0.3 & 0 \\
1 & 0
\end{array}\right], A_{2}=\left[\begin{array}{cc}
0.5447 & 0 \\
1 & 0
\end{array}\right], \\
& A_{3}=\left[\begin{array}{cc}
0.6222 & 0 \\
1 & 0
\end{array}\right], A_{4}=\left[\begin{array}{cc}
0.6265 & 0 \\
1 & 0
\end{array}\right], \\
& A_{5}=\left[\begin{array}{cc}
0.62412 & 0 \\
1 & 0
\end{array}\right], A_{6}=\left[\begin{array}{cc}
0.6255 & 0 \\
1 & 0
\end{array}\right] .
\end{aligned}
$$

Since $A=A_{1} \cdot A_{2} \ldots A_{6}$, then

$$
A=\left[\begin{array}{cc}
0.0248 & 0 \\
0.083 & 0
\end{array}\right] \text {. }
$$

Therefore, by solving the determinant $|A-\lambda I|=0$, then we get $\lambda_{1}=0$, $\lambda_{2}=0.0248$, that is $\left|\lambda_{i}\right|<1$

Which satisfy theorem (2), that is the model have stable limit cycle.

linearization technique, by this technique all nonlinear autoregressive models approximate to linear autoregressive models. This technique consist three steps. 
The existence of a non-zero singular point, the stability of the non-zero singular point and the existence of a limit cycle) if the model have unstable non-zero singular we search for existence of a limit cycles. If the limit cycle exists and stable, then the model is stable otherwise it is unstable. We also give some examples to explain the method.

\section{REFERENCES}

[1] M.B. Priestly (1988)" Nonlinear and Non-stationary Time series Analysis ", Academic Press.

[2] T. Subba Rao, (1981)," On the Theory of Bilinear Time Series Models", Journal of Royal statistical society: series B.

[3] Ozaki, T., and Oda, H.,(1978)," Nonlinear Time Series Model Identification by Akaka's information Criterion, In information and System", ed. Dubuisson. Pergamon Press, Oxford

[4] Tong H. (2011), "Threshold models in time series analysis - 30 years on ", Statistics and Its Interface Volume 4 (2011) 107-118.
[5] Tong, H.,(1990)," Nonlinear Time Series., A dynamical System Approach, Oxford

University Press, New york.

[6] Ozaki, T.(1985)," Nonlinear Time Series Models and Dynamical system", Handbook of statistic ,Vol. 5(ed. Hannan , E.,J. ,and Krishnailah, P., R.,and Rao,M. M.) Elsevier Science Publisher B.,V.,PP(25-83)

[7] Omid S. Fard (2007)" linearization and nonlinear stochastic differential equation with locally Lipchitz condition ", J. of applied mathematics, Vol.1, 2007, no. 52, 2553-2536.

[8] Ozaki, T.,(1982), " The Statistical Analysis of Perturbed Limit Cycle Process Using Nonlinear Time series Models", Journal of Time Series Analysis, Vol.3No.1,PP(29-41).

[9] Chatifield,C.,(2000)," Time series Forecasting ", Chapman and Hall, The University Of Bath, London, UK.

[10] Chatifield,C.,(2004) sixth edition," The Analysis of Time series : An introduction", Chapman and Hall, The University Of Bath, London, UK. 